\title{
Letter to the Editor regarding "Extracorporeal shockwave therapy for the treatment of knee osteoarthritis: a meta-analysis"
}

\author{
Shuxiang $\mathrm{Li}^{1} \cdot$ Kaifeng Zhang ${ }^{1} \cdot$ Xiaoliang Sun ${ }^{1}$
}

Received: 8 February 2020 / Accepted: 19 February 2020/Published online: 29 February 2020

(C) SICOT aisbl 2020

We took great interest in reading the article titled "Extracorporeal shockwave therapy for the treatment of knee osteoarthritis: a meta-analysis" by Hsieh et al. [1]. They performed an interesting meta-analysis on observational studies to investigate the degree of pain reduction and functional outcome after extracorporeal shockwave therapy (ESWT) for knee osteoarthritis. Although we read the article with pleasure, it must be stated that the research has raised some thoughtprovoking issues. Through the present Letter to the Editor, we wish to have this opportunity to comment on this article respectfully.

Firstly, the Methodology stated that the literature search was conducted between 2017 and 2018 without any restriction in language. However, two important studies which were published online in 2013 by Zhao et al. [2] and 2016 by Imamura et al. [3] were missed. This was possibly related to the improper search strategy used in the meta-analysis. Identifying and pooling eligible studies as many as possible are the keys to improving the quality of a meta-analysis. Secondly, for continuous outcomes, each characteristic was calculated using the weighted mean difference. Why not use the standard mean difference? Thirdly, with such high heterogeneities found in all of outcomes (visual analogue scale [VAS] within two weeks, $I^{2}=81 \%$; Western Ontario and McMaster Universities Osteoarthritis Index [WOMAC] within two weeks, $I^{2}=98 \%$; VAS at 4-6 weeks, $I^{2}=93 \%$; WOMAC at four to six weeks, $I^{2}=93 \%$; VAS at six months, $I^{2}=84 \%$; WOMAC at six months, $I^{2}=94 \%$; WOMAC at 12 months, $I^{2}=95 \%$ ), insufficient subgroup analyses and sensitivity analyses had been performed to find the source of heterogeneity. We suggest that the authors should mention the possible

Shuxiang Li and Kaifeng Zhang contributed equally to this work.

Xiaoliang Sun

daw79423@163.com

1 Articular Orthopaedics, The Third Affiliated Hospital of Soochow University, 185 Juqian Road, Changzhou 213003, Jiangsu, China source of heterogeneity in the Discussion. The different study designs may contribute to great clinical heterogeneity; thus, subgroup analysis needs to be conducted on different study designs. Besides, nine studies included in this meta-analysis provided sufficient data to perform a subgroup analysis by type of ESWT (focus or radial) and including the missed study can further help to perform the necessary analysis.

We respectfully appreciate that Hsieh et al. provided us with this important meta-analysis. However, more studies with large sample size and good scientific design should be carried out to clarify this issue.

\section{Compliance with ethical standards}

Competing interests The authors declare that they have no conflict of interest.

Ethical approval This article does not contain any studies with human participants or animals performed by any of the authors.

\section{References}

1. Hsieh CK, Chang CJ, Liu ZW, Tai TW (2020) Extracorporeal shockwave therapy for the treatment of knee osteoarthritis: a meta-analysis. Int Orthop https://doi.org/10.1007/s00264-020-04489-x

2. Zhao Z, Jing R, Shi Z, Zhao B, Ai Q, Xing G (2013) Efficacy of extracorporeal shockwave therapy for knee osteoarthritis: a randomized controlled trial. J Surg Res 185(2):661-666

3. Imamura M, Alamino S, Hsing WT, Alfieri FM, Schmitz C, Battistella LR (2016) Radial extracorporeal shock wave therapy for disabling pain due to severe primary knee osteoarthritis. J Rehabil Med 49(1):54-62

Publisher's note Springer Nature remains neutral with regard to jurisdictional claims in published maps and institutional affiliations. 